\title{
On Monoculture and the Structure of Crop Rotations
}

\author{
David A. Hennessy
}

Working Paper 04-WP 369

August 2004

\author{
Center for Agricultural and Rural Development \\ lowa State University \\ Ames, lowa 50011-1070 \\ www.card.iastate.edu
}

David Hennessy is a professor in the Department of Economics and Center for Agricultural and Rural Development at lowa State University.

The author thanks, without implication, HongLi Feng, Cathy Kling, and Phil Gassman for advice on rotation effects and the related policy environment.

This paper is available online on the CARD Web site: www.card.iastate.edu. Permission is granted to reproduce this information with appropriate attribution to the author.

For questions or comments about the contents of this paper, please contact David Hennessy, 578 Heady Hall, lowa State University, Ames, IA 50011-1070; Ph: 515-294-6171; Fax: 515-294-6336; E-mail: hennessy@iastate.edu.

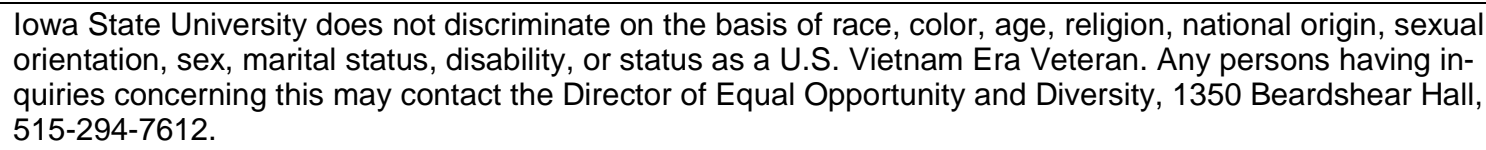
orientation, sex, marital status, disability, or status as a U.S. Vietnam Era Veteran. Any persons having inquiries concerning this may contact the Director of Equal Opportunity and Diversity, 1350 Beardshear Hall, 515-294-7612. 


\begin{abstract}
While rotation strategies are important in determining agricultural commodity supply and environmental benefits from land use, little has been said about the economics of crop rotation. An issue when seeking to identify rotation dominance is whether yield and input-saving carry-over effects persist for one or more years. Focusing on length of carryover, expected profit maximization, and the monoculture decision, this paper develops principles concerning choice of rotation structure. For some rules that we develop, rotations may be discarded without reference to price levels while other rules require price data. We also show how risk aversion in the presence of price uncertainty can alter preferences over rotations. A further consideration in rotation choice is the allocation of time. The problem of crop choice to manage time commitments through the crop year is formally similar to that of crop choice to manage profit risk.
\end{abstract}

Keywords: dominance, jointness, quasiconvexity, rotation algebra, specialization, time rationing.

JEL classification: D2, Q1, Q2 


\section{ON MONOCULTURE AND THE STRUCTURE OF CROP ROTATIONS}

\section{Introduction}

One of the defining features of crop agriculture throughout much of the world is the widespread practice of cropping in rotation. Crop rotations have been practiced since the beginning of agriculture, and some formal rules of thumb are known to have been practiced since medieval times. In order to support mixed farming and to avoid fouling fields, medieval estates in Sussex, England, applied a rotation of wheat, then barley (or oats), then legumes for sheep folding. These estates also grew intensive cereal crops followed by several years of grass (Brandon 1972). Variants of the Dutch/Norfolk system of cereals (wheat, barley, or oats) interspersed with dung-nourished turnips, grass, and legumes to support livestock and replenish the soil were used in much of northern Europe by 1700 (Timmer 1969; Plumb 1952).

Elsewhere in Europe, water was not as plentiful, and fallowing in rotation was the dominant cropping strategy through at least 1700. Newell (1973) and others hold that the replacement of fallow in rotation with forage crops during 1780-1850 was a major contributor to agricultural productivity growth in France by supporting additional animals and enhancing soil fertility. And the introduction of sugar beet to Continental Europe during the Napoleonic wars, to substitute for Caribbean sugarcane, required the practice of rotations of up to seven years (Poggi 1930).

In the United States, too, crop rotation strategies have been an important determinant of regional and crop sector success. Rhode (1995) reports the demise of monoculture wheat in California, eventually to be replaced by more sustainable orchard crops and by horticultural rotations. During the early part of the twentieth century, and partly in response to G.W. Carver's work and advocacy at the Tuskegee Institute, much of the South moved from predominantly monoculture cotton to cotton-based rotations that included peanuts and potatoes. Windish (1981) provides a history of the introduction of the soybean into the Corn Belt, circa 1920. Sugar beet rotations similar to those in Europe were found to be suc- 
cessful in the Upper Midwest (Stilgenbauer 1927). Following the Dust Bowl in the southern Great Plains, the predominant monoculture wheat sequence was replaced by various rotations that often include sorghum and fallow with wheat (Baumhardt 2003).

Miller (2003) has documented growth in specialization on Iowa farms over the period 1880-2000, attributing it largely to technological change with emphasis on scale economies and improved market inputs that substitute for rotation effects. The decline of horsepower, lower costs of trade, and increasing market access have also allowed for increasing regional specialization. Within a region's mainstay crops, however, rotation choice is likely to remain a key determinant of profitability because many motives for use of rotations are likely to persist.

Campbell et al. (1990) provide a list of private motives for using rotations. These include strengthening resistance to soil erosion and soil degradation, improving soil tilth, and also conserving scarce soil moisture. All of these were important motives for Great Plains cropping system adjustments after the Dust Bowl. Soil erosion is among the most serious risks facing global cropland productivity (Pimentel et al. 1995), and land that is not desertified may require additional nutrient inputs to remain productive.

Pests and diseases are important reasons for rotating through potatoes, cereals, and legumes when sugar beet is the primary crop (Poggi 1930; Stilgenbauer 1927; Cai et al. 1997), for rotating soybean with corn (Miller 2003), and for including low-profit oats in wheat-based rotations (Campbell et al. 1990). In the case of sugar beet, nematodes can persist in the field for up to a decade, and nematicide use may not be permitted because of environmental side effects. Even if chemicals can control the problem, the approach introduces the risk of yield loss due to phytotoxic effects. As with the inclusion of soybeans in corn-based rotations, soil fertility can be enhanced by legume production and by incorporating cover crop organic matter residue into the soil. Organic matter also serves to protect the soil from erosion. Forage crops for grazing animals (turnips, or sugar beet tops as a by-product) can be important when seeking to access seasonally high prices and when alternative approaches to conserving feed are costly.

Growers have also expressed direct interest in using rotations because the practice is held to be consistent with sustainability. This has become important beyond the expression of private values or the desire to protect asset value. Public policies in the United 
States and in the European Union provide incentives to promote environmental goals, and market price premia are available for produce known to have been grown in a manner consistent with certain environmental standards.

Risk and cashflow management can also rationalize the use of rotations (Collins and Barry 1986; Froot, Scharfstein, and Stein 1993). While crop prices do have a systematic component, it is not so strong as to marginalize the relevance of a revenue diversification strategy. State contingent markets are available to growers in some countries and for some commodities, while government policies also provide income support. Growers having access to these opportunities do not, however, make the decision to diversify merely to manage risk or stabilize cash flow; they take it as part of a package with rotation effects and other merits.

A further private motive for use of rotations is to better manage labor supply through the year, noted as a problem in monoculture crop agriculture in regions with thin labor markets (Saloutos 1946; Campbell et al. 1990). Soybeans and corn, for example, are sown and harvested over sufficiently distinct periods that growers can better utilize labor, with less reliance on contract sources. Winter and spring variants of wheat and barley also allow for this latitude. Indeed, the significance of seasonal labor constraints in agriculture is borne out by the belief among some historians that it contributed to the nature of industrialization in manufacture (Sokoloff and Dollar 1997) and the pressures toward agricultural mechanization (Musoke and Olmstead 1982; Whatley 1987).

Rotation effects in practiced rotations can also be adverse, at least for some crops in the cycle. Intensive cultivation under one crop may leave compacted soils for the next, while late harvesting may impede preparation for the follow-up planting. Volunteer plants in subsequent years are weeds and may carry disease. Perhaps the strongest adverse effect can be on accounting profit in some rotation years. Some rotation crops, such as oats throughout North America and spring barley in the Palouse region, are almost never grown in monoculture because market prices make it almost impossible to clear a profit over that part of the cycle.

Rotation strategies are of interest to policymakers for a variety of reasons. The public is also concerned about maintaining land quality, while wind-born particles are a health hazard. Siltation of lakes reduces the value of environmental amenities, while siltation of 
reservoirs and rivers require redress through public funds (Wang et al. 2002; Pimentel et al. 1995). The risk and extent of flooding can be reduced by the more varied landscape that exists under diverse cropping (Pimentel et al. 1995). Rotation choices are also seen to alter the use of agricultural chemicals, with attendant consequences for water quality ( $\mathrm{Wu}$ et al. 2004). ${ }^{1}$ Rotations additionally can promote a more diverse ecosystem while reducing reliance on a chemical approach to pest management that may not be either efficient or sustainable (Cowan and Gunby 1996; Batra 1982).

Because of concerns about global warming, participants in agricultural systems around the world may need to address their contributions to greenhouse gas emissions. The United States emitted about 1,580 million metric tons of $\mathrm{CO}_{2}$ in 2001, while Lal et al. (1999) estimate that the use of improved crop rotations and winter cover crops can mitigate this amount to the extent of about 5-15 million metric tons. When compared with afforestation, this approach is a low-cost approach to sequestration (but with limited sequestration potential) (Lewandrowski et al. 2004).

Agricultural commodity policies inevitably have indirect implications for rotation strategies, but more recent policies in the United States and European Union have more directly targeted rotation strategies. Agri-environmental schemes were institutionalized in E.U. rules following the 1992 Common Agricultural Policy reforms. While implementation varies across countries, subsidies are commonly provided to encourage integrated farming practices that require less intensive use of market inputs, to facilitate the switch to organic farming, and to promote a picturesque landscape. The U.S. Food, Agricultural, Conservation, and Trade Act of 1990 provided funds to subsidize farm production practices that are not harmful to water quality. The 1996 U.S. farm bill extended the approach by funding the Environmental Quality Incentives Program (EQIP) to subsidize voluntary conservation activities by farmers and ranchers. While the practices subsidized vary across the country, a targeted practice standard to be subsidized is that of conservation crop rotation in which a repeated sequence of crops is considered to promote environmental goals. Commencing in 2004, a separate program that focuses on specific watersheds, called the Conservation Security Program, provides funds to entice growers into contracts that limit growing activities. Among the constraints are rotation restrictions that emphasize perennial crops in rotation. 
Given the prevalence of rotations in global crop agriculture, a better understanding of the economics of rotation choices should prove to be very useful for commodity policy analysts. It should also be useful when analyzing the environmental economics of soil, water, rural amenities, and global warming. The advent of spatial information collection and allied techniques, such as global positioning technologies, the Erosion/Productivity Impact Calculator (Sharpley and Williams 1990), and the U.S. National Resources Inventory data, allow for spatial analysis of likely and actual policy consequences. Newer technologies may also permit better monitoring of agricultural production practices. Thus, the need for an economic understanding of rotation choices is strong both to provide insights and to guide policy implementation. Yet research on the economics of crop rotation is quite limited.

Linear programming techniques were quickly adapted to accommodate crop rotation effects (Koopmans 1951). While programming provides the means for empirical analysis, the framework does not appear to have been used to identify conceptual insights on the structure of rotations. Realizing that an understanding of dynamic interactions in dual analysis was needed to appreciate the role of incentives in such matter as soil capital formation, Chambers and Lichtenberg (1995), Färe and Grosskopf (1996), and others have developed empirically implementable dynamic models of production. Jaenicke (2000) has applied the approach, providing evidence in favor of the claim that soil capital matters for corn and soybean production in Rodale, Pennsylvania. Thomas (2003) has implemented a model in which carry-over effects can be estimated using farm choices and in which the optimality of rotations can be tested.

Stepping back from identifying rotation effects, the intent of the present paper is to ask what the consequences of given rotation effects are. Because the possible motives for rotation choice are many and interconnected, no single article could provide a comprehensive analysis. We confine attention to three general effects where the gains from specialization are opposed by some incentive to spread land across a variety of uses. We develop first a conceptual approach to identifying dominated rotations under input and output carry-over effects in the absence of risk aversion, and we identify rules of thumb for eliminating rotations. Under one-year rotation effects, the glue-on principle screens out the use of rotations by comparison with embedded rotations while the insert principle 
discards rotations involving immediate replications. These effects are purely structural, and neither relies on prices.

Under multi-year rotation effects, the sunk cost principle explores the roles of fertility accumulation and switching costs on length and composition of rotation. Working with rotations that have arbitrary rotation effects, the specialization principle invokes quasiconvexity in the objective function when seeking to maximize expected profit across rotation choices to identify the private optimality of monoculture. Both of these effects are price-dependent. The switching principle, which is price-independent, eliminates rotations relative to permuted rotations.

The second and third general effects that are studied concern gains from diversification in the presence of conditions that predispose solutions toward the interior. Under risk aversion, much of the earlier analysis carries through but with some qualifications. Since linearity is broken, rotation and monoculture strategies may be mixed in an optimal land allocation. Labor use diversification is also an issue when rural labor markets are thin. Extending tools used in the analysis of risk preference effects, we model the extent of systemic correlations in demand for time across crops to identify when monoculture might apply. Neither effect necessarily requires crop rotations to rationalize diversification because one can diversify by growing a portfolio of crops sown under monoculture. But if rotation effects are present, then risk and labor diversification effects can tip the balance away from monoculture. The paper concludes with some thoughts on further work in the area.

\section{Concepts}

One acre of land may be allocated to any among $m$ crops, each of which uses the land for one year. ${ }^{2}$ The crops are labeled $u_{i}, i \in\{1,2, \ldots, m\}=\Omega_{m}$. A monoculture rotation using crop $u_{i}$ is labeled as $\left\langle u_{i}\right\rangle$. A rotation $R$ using $u_{i_{1}}$ and then $u_{i_{2}}$ and so on through $u_{\hat{i}}$ is labeled as $R=\left\langle\begin{array}{llll}u_{i_{1}} u_{i_{2}} & \ldots & u_{\hat{i}}\end{array}\right.$. If $u_{k}$ is an entry in $\left\langle u_{i_{1}} u_{i_{2}} \ldots u_{\hat{i}}\right\rangle$ then $u_{k}$ is said to be in $R, u_{k} \in R$, and we say that $u_{k}$ is a letter in the rotation. An adjoining set of letters in a rotation is referred to as a sequence, that is, $u_{\hat{i}} u_{i_{1}} u_{i_{2}}$ is a three-letter sequence in $R$ where we have used the fact that the rotation is seamless and so 
$\left\langle\begin{array}{lllll}u_{i_{1}} u_{i_{2}} & \cdots & u_{\hat{i}}\end{array}\right\rangle=\left\langle\begin{array}{lllll}u_{i_{2}} & \ldots & u_{\hat{i}} u_{i_{1}}\end{array}\right\rangle$. Throughout, we will denote a rotation by the least sequence length before repetition, that is, $\left\langle u_{1} u_{2}\right\rangle$ and not $\left\langle u_{1} u_{2} u_{1} u_{2}\right\rangle$.

The planting time expectation of harvest time output prices are exogenous to the farm at $P_{i}, i \in \Omega_{m}$, and these prices are fixed over time. Absent rotation effects, the output yields are $q_{i}, i \in \Omega_{m}$. Absent rotation effects, the quantities of inputs used are $x_{i}, i \in \Omega_{s}$, and all can be purchased in competitive markets at respective prices $w_{i}, i \in \Omega_{s}$. Concatenating price vectors, the vector of all these prices is written as $r$ with arguments $r_{1}$ through $r_{h}=r_{m+s}$ where the first $m$ arguments are output prices. The associated netput vector, with outputs listed first, is $z$. Absent any consideration of rotation effects, the profit from producing the $u_{i}$ crop is $\pi^{u_{i}}(r), i \in \Omega_{m}$.

Rotation effects alter these profits, creating a jointness in production. We model rotation effects as a location mapping in input-output space. For example, whereas the production function in a continuous corn rotation with applied nitrogen as the sole input might have been $q_{c}=f(x)$, in a corn-after-soybean rotation it becomes $q_{c}=f(x)+5$ because of a five-bushel yield boost due to rotation. Or the production function might become $q_{c}=f(x+10)+5$ where there is a $10 \mathrm{lb} /$ acre nitrogen savings in addition to the five-bushel yield boost. For interior input choice solutions-and we make this assumption throughout the paper-profit that an accountant ignoring rotation effects might attribute to corn increases by $5 P_{\text {corn }}+10 w_{\text {nitrogen }}$ due to rotation effects. As we will show, whether these spillover yield and input effects persist for one or more years into the future is important in determining what one can relate about the optimality of a particular rotation. We will develop our analysis first when spillover yield and input effects persist for one year (one-year memory), deferring the general case to later.

\section{One-Year Memory}

If the $u_{i}$ crop is followed by the $u_{j}$ crop in some rotation labeled $R$, then use the rotation-conditioned year lag operation $L(\cdot ; R)$ to label $u_{i}=L\left(u_{j} ; R\right)$. The spillover effect 
regarding $z_{t}, t \in \Omega_{h}$, is written as $\alpha_{u_{j} \mid L\left(u_{j} ; R\right)}^{t}$. The one-year memory is reflected in the fact that the effect does not depend upon crops before $u_{i}$ in the rotation. A natural restriction applies to these $\alpha_{u_{j} \mid L\left(u_{j} ; R\right)}^{t}$. When $t \in \Omega_{m}$ and $t \neq j$ then $\alpha_{u_{j} \mid L\left(u_{j} ; R\right)}^{t}=0$ regardless of rotation. This restriction is merely to assert that wheat is not harvested in the year that alfalfa is grown in a wheat-alfalfa rotation.

Accounting profit for the $u_{j}$ crop following the $u_{i}$ crop in rotation $R$ is

$$
\pi^{u_{j}}(r)+\sum_{t=1}^{h} \alpha_{u_{j} \mid L\left(u_{j} ; R\right)}^{t} r_{t} ; \quad u_{i}=L\left(u_{j} ; R\right)
$$

Average profit per year over the whole rotation is

$$
V(R)=\frac{1}{|R|} \sum_{u_{j} \in R}\left(\pi^{u_{j}}(r)+\sum_{t=1}^{h} \alpha_{u_{j} \mid L\left(u_{j} ; R\right)}^{t} r_{t}\right),
$$

where $|R|$ is rotation length (years before rotation repeats) and $\sum_{u_{j} \in R}$ sums across each letter entry in the sequence representing $R$. In this section we use equation (2) to ask, When can a rotation be ruled out given the availability of structurally similar but simpler rotations?

\section{Glue-On Principle}

Some consideration of the structure of a rotation identifies situations in which one can dispense with prices and yet remove a rotation from the relevant decision set. The main insight can be obtained upon making graphical depictions of two rotations. The left side of Figure 1 shows rotations $R_{1}=\left\langle u_{1} u_{2} u_{3}\right\rangle$ and $R_{2}=\left\langle u_{1} u_{2} u_{4} u_{5}\right\rangle=\left\langle u_{2} u_{4} u_{5} u_{1}\right\rangle$. The time sequences of both rotations are to be read clockwise. The dotted curve segments show where new bonding will occur and the vertical bars show where bonds will be broken to allow for new bonds. Now consider rotation $R_{3}=\left\langle u_{1} u_{2} u_{4} u_{5} u_{1} u_{2} u_{3}\right\rangle$, on the right side of Figure 1. This rotation is constructed from the simpler rotations by cutting both loops $R_{1}$

and $R_{2}$ between $u_{1}$ and $u_{2}$, then adjoining the $u_{1}$ end from $R_{1}$ to the $u_{2}$ end from $R_{2}$ and the $u_{1}$ end from $R_{2}$ to the $u_{2}$ end from $R_{1}$. In order to see the relevance of this glue-on, a definition is needed. 

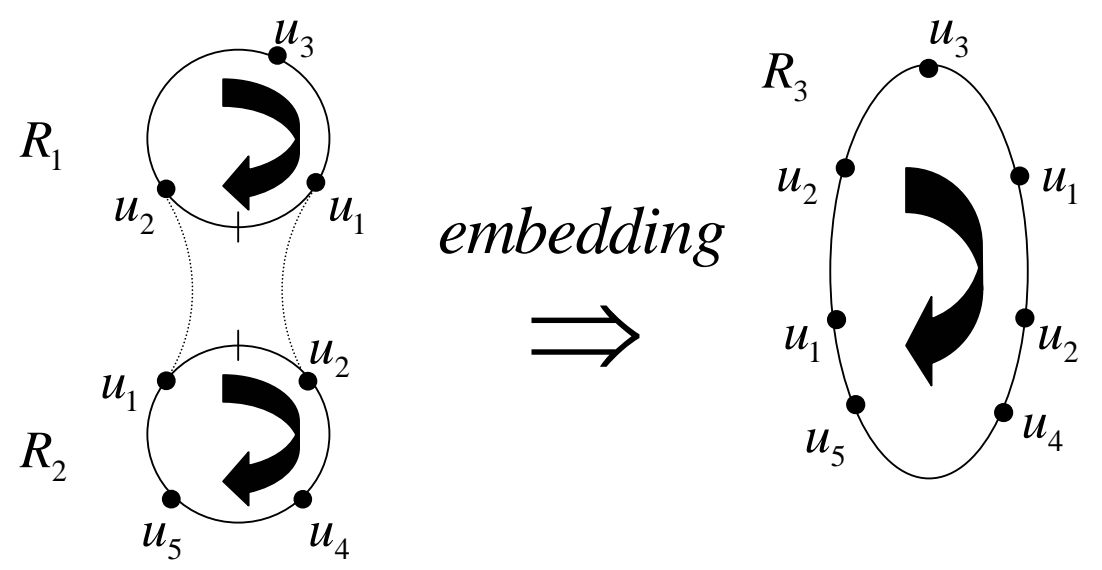

FIGURE 1. Rotation loops $\boldsymbol{R}_{1}$ and $\boldsymbol{R}_{\mathbf{2}}$ are joined to make $\boldsymbol{R}_{3}$

DEFINITION 1. Rotations $R_{1}$ and $R_{2}$ are said to be embedded in rotation $R_{3}$ if

(a) $R_{1}$ and $R_{2}$ contain a common letter sequence $u_{i} u_{j} ; i, j \in \Omega_{m}$, while

(b) $R_{3}$ satisfies $\left|R_{3}\right|=\left|R_{1}\right|+\left|R_{2}\right|$ and contains all the letters in each of $R_{1}$ and $R_{2}$ in sequence, commencing at $u_{j}$.

Part (a) allows for the embedding to occur by breaking both rotations between $x_{i}$ and $x_{j}$. Part (b) ensures that the embedding does occur and that no surplus letters are introduced. Profits for rotations $R_{i}, i \in\{1,2\}$, to be embedded are

$$
V\left(R_{i}\right)=\frac{1}{\left|R_{i}\right|} \sum_{u_{k} \in R_{i}}\left(\pi^{u_{k}}(r)+\sum_{t=1}^{h} \alpha_{u_{k} \mid L\left(u_{k} ; R_{i}\right)}^{t} r_{t}\right) .
$$

Take a weighted sum to obtain

$$
\begin{aligned}
& \frac{\left|R_{1}\right|}{\left|R_{3}\right|} V\left(R_{1}\right)+\frac{\left|R_{2}\right|}{\left|R_{3}\right|} V\left(R_{2}\right) \\
& =\frac{1}{\left|R_{3}\right|} \sum_{u_{k} \in R_{1}}\left(\pi^{u_{k}}(r)+\sum_{t=1}^{h} \alpha_{u_{k} \mid L\left(u_{k} ; R_{3}\right)}^{t} r_{t}\right)+\frac{1}{\left|R_{3}\right|} \sum_{u_{k} \in R_{2}}\left(\pi^{u_{k}}(r)+\sum_{t=1}^{h} \alpha_{u_{k} \mid L\left(u_{k} ; R_{3}\right)}^{t} r_{t}\right) \\
& =\frac{1}{\left|R_{3}\right|} V\left(R_{3}\right) .
\end{aligned}
$$


Definition 1, parts (a) and (b), allows us to adapt the histories for $R_{1}$ and $R_{2}$ to that for $R_{3}$ without losing step. The connections are seamless. But

$$
\frac{\left|R_{1}\right|}{\left|R_{3}\right|} V\left(R_{1}\right)+\frac{\left|R_{2}\right|}{\left|R_{3}\right|} V\left(R_{2}\right) \leq \max \left\{V\left(R_{1}\right), V\left(R_{2}\right)\right\},
$$

so that the embedding rotation can have profit no larger than the largest among those of the embedded rotations.

RESULT 1. (Glue-on principle) Under one-year memory, if $R_{1}$ and $R_{2}$ are embedded in $R_{3}$ then $R_{3}$ is weakly dominated by either $R_{1}$ or $R_{2}$.

EXAMPLE 1. Camara, Young, and Hinman (1999) provide case studies of six three-year crop rotations used in the Palouse region of Washington and Idaho states. Each involves winter wheat and at least one other cereal, while five also involve a legume (peas or lentils). Case study farms $\mathrm{A}$ and $\mathrm{B}$ use winter wheat $(W w)$ then spring barley $(S b)$ then peas $(P e)$ and $W w$ then $S b$ then spring wheat $(S w)$. If $R_{1}=\langle W w S b P e\rangle, R_{2}=\langle W w S b S w\rangle$, and $R_{3}=\langle W w S b P e W w S b S w\rangle$, then $R_{3}$ can be removed from consideration under one-year memory. Heady (1951) reports a field trial in Ohio with rotation Corn-Corn-WheatAlfalfa-Alfalfa, which is an embedding of Corn-Alfalfa and Corn-Wheat-Alfalfa, and so can be ruled out because of Result 1 under one-year memory. Campbell et al. (1990) report field trials that involve Fallow-Wheat-Wheat-Hay-Hay-Hay, where again Result 1 and one-year memory identify domination.

\section{Duplicate Insertion Principle}

Application of Definition 1 requires a sequence of two letters common to two rotations. Sometimes the idea of common sequences does not apply but the mechanism used to bond the two rotations remains relevant. When two rotations differ only by the inclusion of a repeated letter then the repetition creates a redundancy in the conditions specified in Definition 1. 
DEFINITION 2. If $u_{i} \in R_{1}$ and $u_{i} u_{i}$ replaces a $u_{i}$ in $R_{1}$ to generate rotation $R_{2}$ (so that $\left.\left|R_{2}\right|=\left|R_{1}\right|+1\right)$ then $R_{2}$ is said to be obtained from $R_{1}$ by duplicate insertion.

With one-year memory, if a $u_{i}$ is snipped out of $R_{2}$ then the remaining $u_{i}$ bonds just as well as in $R_{2}$. Denoting a sequence $u_{i} u_{i}$ as $U$, suppose that $u_{k}$ precedes $u_{i} u_{i}$ in $R_{2}$ so that $^{3}$

$$
\begin{aligned}
& V\left(R_{2}\right)=\frac{1}{\left|R_{2}\right|} \sum_{\substack{u_{j} \in R_{2} \\
u_{j} \in U}}\left(\pi^{u_{j}}(r)+\sum_{t=1}^{h} \alpha_{u_{j} \mid L\left(u_{j} ; R_{2}\right)}^{t} r_{t}\right)+\frac{2 \pi^{u_{i}}(r)+\sum_{t=1}^{h} \alpha_{u_{i} \mid u_{i}}^{t} r_{t}+\sum_{t=1}^{h} \alpha_{u_{i} \mid u_{k}}^{t} r_{t}}{\left|R_{2}\right|} \\
& =\frac{\left(\left|R_{2}\right|-1\right)}{\left|R_{2}\right|} \frac{1}{\left(\left|R_{2}\right|-1\right)} \sum_{u_{j} \in R_{1}}\left(\pi^{u_{j}}(r)+\sum_{t=1}^{h} \alpha_{u_{j} \mid L\left(u_{j} ; R_{1}\right)}^{t} r_{t}\right)+\frac{\pi^{u_{i}}(r)+\sum_{t=1}^{h} \alpha_{u_{i} \mid u_{i}}^{t} r_{t}}{\left|R_{2}\right|} \\
& =\frac{\left(\left|R_{2}\right|-1\right)}{\left|R_{2}\right|} V\left(R_{1}\right)+\frac{1}{\left|R_{2}\right|} V\left(\left\langle u_{i}\right\rangle\right) \leq \max \left\{V\left(R_{1}\right), V\left(\left\langle u_{i}\right\rangle\right)\right\} .
\end{aligned}
$$

This observation allows us to assert the following.

RESULT 2. (Duplicate insertion principle) Under one-year memory, if $R_{2}$ is obtained from $R_{1}$ by duplicate insertion of $u_{i}$ then $R_{2}$ is weakly dominated by either $R_{1}$ or $\left\langle u_{i}\right\rangle$.

EXAMPLE 2. Compare the two-crop corn-soybean rotation, $\langle C S\rangle$, with alternatives also sometimes used in the U.S. Corn Belt, $\langle C C S\rangle$ and $\langle C C C S\rangle$. If one-year memory applies then Result 2 precludes both $\langle C C S\rangle$ and $\langle C C C S\rangle$. Duplicate inserts also exist in the Example 1 rotations from Heady (1951) and Campbell et al. (1990), so one may wonder whether one principle is subsumed. While Results 1 and 2 are strongly related, Result 1 would not preclude the extended rotations $\langle C C S\rangle$ and $\langle C C C S\rangle$. Similarly, Result 2 could not be used to rule out $R_{3}$ in Example 1.

\section{N-Year Memory}

The main, tedious, and important distinction between $\mathrm{N}$-year memory and one-year memory is that operator $L(; R)$ is no longer dependent only on the last chosen crop but 
rather on the last $\mathrm{N}$ crops. Results 1 and 2 adapt readily to the $\mathrm{N}$-year memory context, and indeed to contexts where length of memory depends on the crop sequence. ${ }^{4}$ But the ability to identify dominance will typically be weakened. In what follows we illustrate with two-year memory only. Write $\alpha_{u_{i} \mid u_{j} u_{k}}^{t}$ for the netput $t$ rotation effects when growing crop $u_{i}$ given that the immediate predecessor crop was $u_{j}$ and $u_{k}$ preceded that.

EXAMPLE 3. Under two-year memory with yield effects only, consider the three rotations $\langle C S\rangle,\langle C C S\rangle$, and $\langle C C C S\rangle$. The last two rotations could be discarded under one-year memory by use of the duplicate insertion principle. Under two-year memory,

$$
\begin{aligned}
& V(\langle C S\rangle)=\frac{1}{2}\left(\pi^{C}(r)+\pi^{S}(r)+\alpha_{C \mid S C}^{C} P_{C}+\alpha_{S \mid C S}^{S} P_{S}\right) \\
& V(\langle C C S\rangle)=\frac{1}{3}\left(2 \pi^{C}(r)+\pi^{S}(r)+\alpha_{C \mid S C}^{C} P_{C}+\alpha_{C \mid C S}^{C} P_{C}+\alpha_{S \mid C C}^{S} P_{S}\right) \\
& V(\langle C C C S\rangle)=\frac{1}{4} V(\langle C\rangle)+\frac{3}{4} V(\langle C C S\rangle) .
\end{aligned}
$$

Rotation $\langle C C C S\rangle$ can be discarded because Result 2 applies under two-year memory if duplicate insertion is replaced by the idea of inserting a third consecutive year of the crop when the sequence had been just two consecutive years. But $\langle C C S\rangle$ can dominate $\langle C S\rangle$ and $\langle C\rangle$ under appropriate carry-over and price parameters. To verify this, assume that $\pi^{C}(r)=\pi^{S}(r)$ so that $V(\langle C C S\rangle)>\max \{V(\langle C\rangle), V(\langle C S\rangle)\}$ whenever

$$
\alpha_{S \mid C C}^{S} P_{S}>\left(3 \alpha_{C \mid C C}^{C}-\alpha_{C \mid S C}^{C}-\alpha_{C \mid C S}^{C}\right) P_{C} ; \quad\left(2 \alpha_{C \mid C S}^{C}-\alpha_{C \mid S C}^{C}\right) P_{C}>\left(3 \alpha_{S \mid C S}^{S}-2 \alpha_{S \mid C C}^{S}\right) P_{S}
$$

Choices of $\alpha$ parameters and prices are readily identified such that both inequalities are satisfied.

EXAMPLE 4. To show how involved price interactions can be under two-year memory, consider a three-crop rotation of length four. Crops $A$ and $C$ are grown once while crop $B$ is grown twice. There are three such rotations: $\langle A B B C\rangle,\langle A B C B\rangle$, and $\langle A C B B\rangle$. For two-year memory and yield effects only, we seek to establish the maximum value among 


$$
\begin{aligned}
& V(\langle A B B C\rangle)=\frac{1}{4}\left(\pi^{A}(r)+2 \pi^{B}(r)+\pi^{C}(r)+\alpha_{A \mid C B}^{A} P_{A}+\alpha_{B \mid A C}^{B} P_{B}+\alpha_{B \mid B A}^{B} P_{B}+\alpha_{C \mid B B}^{C} P_{C}\right) \\
& V(\langle A B C B\rangle)=\frac{1}{4}\left(\pi^{A}(r)+2 \pi^{B}(r)+\pi^{C}(r)+\alpha_{A \mid B C}^{A} P_{A}+\alpha_{B \mid A B}^{B} P_{B}+\alpha_{B \mid C B}^{B} P_{B}+\alpha_{C \mid B A}^{C} P_{C}\right) \\
& V(\langle A C B B\rangle)=\frac{1}{4}\left(\pi^{A}(r)+2 \pi^{B}(r)+\pi^{C}(r)+\alpha_{A \mid B B}^{A} P_{A}+\alpha_{B \mid C A}^{B} P_{B}+\alpha_{B \mid B C}^{B} P_{B}+\alpha_{C \mid A B}^{C} P_{C}\right) .
\end{aligned}
$$

Because of profit function homogeneity, we may arbitrarily normalize one price without loss of insight. Let $P_{C}=1$ so that the break-even lines are

$\langle A B B C\rangle,\langle A B C B\rangle: \quad\left(\alpha_{A \mid C B}^{A}-\alpha_{A \mid B C}^{A}\right) P_{A}+\left(\alpha_{B \mid A C}^{B}+\alpha_{B \mid B A}^{B}-\alpha_{B \mid A B}^{B}-\alpha_{B \mid C B}^{B}\right) P_{B}=\alpha_{C \mid B A}^{C}-\alpha_{C \mid B B}^{C} ;$

$\langle A B B C\rangle,\langle A C B B\rangle: \quad\left(\alpha_{A \mid C B}^{A}-\alpha_{A \mid B B}^{A}\right) P_{A}+\left(\alpha_{B \mid A C}^{B}+\alpha_{B \mid B A}^{B}-\alpha_{B \mid C A}^{B}-\alpha_{B \mid B C}^{B}\right) P_{B}=\alpha_{C \mid A B}^{C}-\alpha_{C \mid B B}^{C} ;$

$$
\langle A B C B\rangle,\langle A C B B\rangle: \quad\left(\alpha_{A \mid B C}^{A}-\alpha_{A \mid B B}^{A}\right) P_{A}+\left(\alpha_{B \mid A B}^{B}+\alpha_{B \mid C B}^{B}-\alpha_{B \mid C A}^{B}-\alpha_{B \mid B C}^{B}\right) P_{B}=\alpha_{C \mid A B}^{C}-\alpha_{C \mid B A}^{C} .
$$

They are congruent: subtract the first from the second to obtain the third so that any solution to the first two also satisfies the third. With $\alpha_{C \mid B A}^{C}-\alpha_{C \mid B B}^{C}=1=\alpha_{C \mid A B}^{C}-\alpha_{C \mid B B}^{C}$,

$\alpha_{A \mid C B}^{A}=\alpha_{A \mid B B}^{A}, \alpha_{A \mid B C}^{A}-\alpha_{A \mid B B}^{A}=-1, \alpha_{B \mid A C}^{B}+\alpha_{B \mid B A}^{B}-\alpha_{B \mid C A}^{B}-\alpha_{B \mid B C}^{B}=1$, and

$\alpha_{B \mid A C}^{B}+\alpha_{B \mid B A}^{B}=\alpha_{B \mid A B}^{B}+\alpha_{B \mid C B}^{B}$, then $V(\langle A B B C\rangle)=V(\langle A B C B\rangle)$ implies $P_{A}=1$,

$V(\langle A B B C\rangle)=V(\langle A C B B\rangle)$ implies $P_{B}=1$, and

$V(\langle A B C B\rangle)=V(\langle A C B B\rangle)$ implies $P_{B}=P_{A}$. When $P_{A} \geq 1$ and $P_{B} \geq 1$, then $\langle A B B C\rangle$

dominates (weakly). When $P_{A} \leq 1$ and $P_{B} \geq P_{A}$, then $\langle A B C B\rangle$ dominates (weakly). The

situation is depicted in Figure 2. These preferences are driven entirely by the imposed carry-over effects, and one could choose carry-over parameter values such that preferred rotations on these three regions in output price space were interchanged.

EXAMPLE 5. (Sunk cost principle) As with the Sussex systems and some systems reported in Example 1, pasture and other perennial crops often enter a rotation. These crops may involve start-up (switching) costs because of low productivity in the first year, and switching costs will affect rotation structure. Suppose that crop $A$ is perennial (pasture, alfalfa, etc.) while crop $B$ is annual. Start-up costs for the crop amount to $K \geq 0$. There are no rotation effects concerning the productivity of $\operatorname{crop} A$, but there is a multi-year productivity effect under crop $B$. Specifically, the first year of crop $B$ production after 


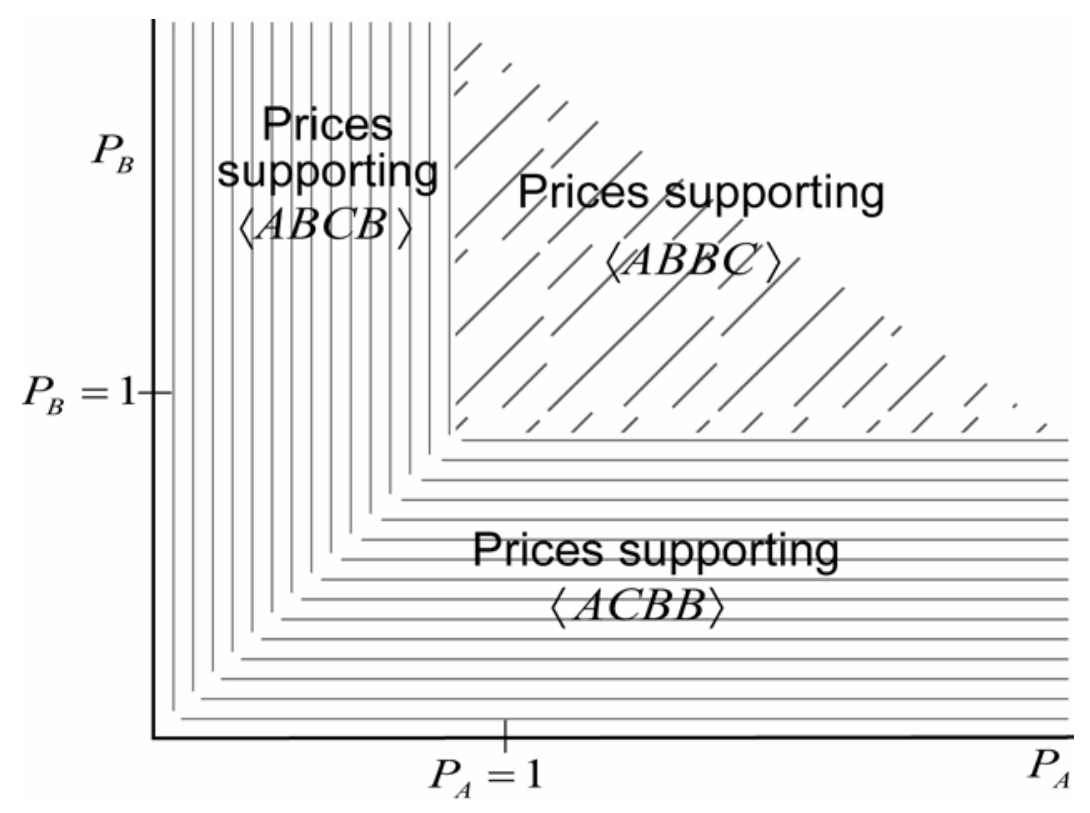

FIGURE 2. Choosing among permuted rotations

$N-1$ years of crop $A$ receives $f(N)$ additional yield, $f(1)=0$, but the second and subsequent years receive no additional yield. The rotation effect, $f(N)$, is increasing but at a decreasing rate while $f(N)$ is also bounded. Baseline crop $A$ profit is $\pi^{A}$ while baseline crop $B$ profit is $\pi^{B}$, with $\pi^{A}>\pi^{B}$ so that $A$ would be the preferred crop absent rotation effects.

Write the rotation where $N-1$ years of crop $A$ are followed by one year of crop $B$, before the rotation starts over, as $\left\langle A^{N-1} B\right\rangle$. The rotation has value

$$
V\left(\left\langle A^{N-1} B\right\rangle\right)=\frac{(N-1) \pi^{A}+\pi^{B}+f(N) P_{B}-K}{N} .
$$

Differentiate, with the order indicated by the number of prime symbols, to obtain value

$$
\frac{d V\left(\left\langle A^{N-1} B\right\rangle\right)}{d N}=\frac{\pi^{A}-\pi^{B}+K-f(N) P_{B}}{N^{2}}+\frac{f^{\prime}(N) P_{B}}{N}=\frac{\pi^{A}-V\left(\left\langle A^{N-1} B\right\rangle\right)}{N}+\frac{f^{\prime}(N) P_{B}}{N} .
$$

The first fraction at right must be negative for some positive natural number $N$ if rotation $V\left(\left\langle A^{N-1} B\right\rangle\right)$ is to be chosen over specialization in $A$. Given $\pi^{A}>\pi^{B}$, this means that 
$K<f(N) P_{B}$ is required; otherwise rotation-effect gains in crop $B$ would not outweigh the switching costs. The set of admissible $N$ are the positive natural numbers satisfying $\{N$ : $\left.\pi^{A}-\pi^{B}+K-f(N) P_{B}<0\right\}$. If the set is empty then there will never be an incentive to grow $B$ in rotation. The set contracts as $K$ increases and, for given price levels, there exists a ceiling value of $K$ above which $\langle A\rangle$ is preferred. The second fraction in (12) is positive, and represents the marginal revenue from increased fertility in $B$ when averaged over all rotation years.

A second differentiation gives

$$
\frac{d^{2} V\left(\left\langle A^{N-1} B\right\rangle\right)}{d N^{2}}=\frac{2\left(\pi^{B}-\pi^{A}-K+f(N) P_{B}\right)}{N^{3}}+\frac{f^{\prime \prime}(N) P_{B}}{N}-2 \frac{f^{\prime}(N) P_{B}}{N^{2}} .
$$

This is negative at any point satisfying $d V\left(\left\langle A^{N-1} B\right\rangle\right) / d N=0$, so that local concavity applies. It is readily demonstrated that optimum $N$ increases with the level of $K$. Crop $A$ becomes more prominent in the rotation as the start-up cost for crop $A$ increases because $\pi^{A}>\pi^{B}$. The only motive for choosing $B$ is the rotation effect so the start-up cost is attributed to crop $B$ rather than crop $A$.

\section{General Analysis of Rotation Effects}

Memory structure is not necessary for some conclusions to be made on optimal rotation choices. Two are what we call the specialization principle and the switching principle. In addition, we comment on the role of price homogeneity on the structure of rotation choices and how subsidies can affect that structure.

\section{Specialization Principle}

Monoculture is largely about gains from specialization. These gains can come in many forms, including the consequences of stronger incentives to develop crop-specific human capital. The sort of specialization we consider here is not in any way dynamic. It refers to the circumstances under which rotation effects are insufficient to dominate the discretion to specialize in one particular crop. Write the maximum among monoculture profits for crops in rotation $R$ as 


$$
\mathscr{L}(R)=\max _{u_{i} \in R}\left\{V\left(\left\langle u_{i}\right\rangle\right)\right\}
$$

By the convexity and symmetry of the $\max \{\cdot\}$ function in (14), an application of Jensen's inequality provides 5

$$
\mathscr{L}(R)=\max _{u_{i} \in R}\left\{V\left(\left\langle u_{i}\right\rangle\right)\right\} \geq \frac{1}{|R|} \sum_{u_{j} \in R}\left(\pi^{u_{j}}(r)+\sum_{t=1}^{h} \alpha_{u_{j} \mid u_{j}}^{t} r_{t}\right) \equiv \hat{\mathscr{L}}(R) .
$$

Specialization will certainly be preferred if the value $\hat{\mathscr{L}}(R)$ defined in (15) exceeds $V(R)$.

Taking the difference, $\Delta(R)=\hat{\mathscr{L}}(R)-V(R)$, we obtain

$$
\Delta(R)=\frac{1}{|R|} \sum_{u_{j} \in R} \sum_{t=1}^{h}\left(\alpha_{u_{j} \mid u_{j}}^{t}-\alpha_{u_{j} \mid L\left(u_{j} ; R\right)}^{t}\right) r_{t}=\sum_{t=1}^{h} r_{t} \bar{\delta}^{t}(R),
$$

where $\bar{\delta}^{t}(R)=(|R|)^{-1} \sum_{u_{j} \in R}\left(\alpha_{u_{j} \mid u_{j}}^{t}-\alpha_{u_{j} \mid L\left(u_{j} ; R\right)}^{t}\right)$. The inference from the comparison may be summarized as follows.

RESULT 3. (Separation principle) If $\sum_{t=1}^{h} r_{t} \bar{\delta}^{t}(R)>0$ then rotation $R$ is dominated by a monoculture rotation.

Of course, the comparison cannot relate which crop, were it grown in monoculture, would dominate the rotation.

EXAMPLE 6. For the two-crop corn-soybean rotation, $\langle C S\rangle$ with $C$ for corn, let $\alpha_{C \mid S}^{t}=\alpha_{S \mid C}^{t}=\alpha_{C \mid C}^{t}=\alpha_{S \mid S}^{t}=0$ for all inputs. In addition, restrictions $\alpha_{C \mid S}^{S}=\alpha_{S \mid C}^{C}=0$ apply. With $P_{C}$ and $P_{S}$ as output prices, then $\bar{\delta}^{C}(\langle C S\rangle)=0.5\left(\alpha_{C \mid C}^{C}-\alpha_{C \mid S}^{C}\right)$ and $\bar{\delta}^{S}(\langle C S\rangle)=0.5\left(\alpha_{S \mid S}^{S}-\alpha_{S \mid C}^{S}\right)$. Upon applying the normalization $\alpha_{C \mid C}^{C}=\alpha_{S \mid S}^{S}=0$, which only means that monoculture rotations are taken as the baseline for comparison, then $\sum_{t=1}^{h} r_{t} \bar{\delta}^{t}(\langle C S\rangle)=-\alpha_{C \mid S}^{C} P_{C}-\alpha_{S \mid C}^{S} P_{S}$. If $\alpha_{C \mid S}^{C} \geq 0$ and $\alpha_{S \mid C}^{S} \geq 0$ under one-year memory, then the separation principle certainly does not apply so that one is no wiser on the admissibility of the rotation. If either of the carry-over yield effects is negative, then there exist 
output price vectors such that the principle does apply and $\langle C S\rangle$ can be ruled out at these price vector evaluations.

\section{Switching Principle}

Consider two rotations $R_{1}$ and $R_{2}$, that differ only by permutation. For example, let $R_{1}=\left\langle u_{1} u_{2} u_{3} u_{4}\right\rangle$ and $R_{2}=\left\langle u_{1} u_{4} u_{3} u_{2}\right\rangle$. The rotations are the same up to transposition $u_{2} \leftrightarrow u_{4}$. The general expression for $V\left(R_{2}\right)-V\left(R_{1}\right)$ is

$$
V\left(R_{2}\right)-V\left(R_{1}\right)=\frac{1}{\left|R_{1}\right|} \sum_{t=1}^{h} r_{t} \sum_{u_{j} \in R_{1}}\left(\alpha_{u_{j} \mid L\left(u_{j} ; R_{2}\right)}^{t}-\alpha_{u_{j} \mid L\left(u_{j} ; R_{1}\right)}^{t}\right)
$$

where baseline profit disappears upon taking differences under the permutation attribute. An implication is Result 4.

RESULT 4. (Convex cone property) Let $R_{1}$ and $R_{2}$ differ only by permutation. If $V\left(R_{2}\right)>V\left(R_{1}\right)$ at price vector $r^{\prime} \in \overline{\mathbb{R}}_{+}^{h}$ and also at price vector $r^{\prime \prime} \in \overline{\mathbb{R}}_{+}^{h}$, then $V\left(R_{2}\right)>V\left(R_{1}\right)$ at any price vector $\lambda r^{\prime}+(1-\lambda) r^{\prime \prime}, \lambda \in[0,1]$, any price vector $\lambda r^{\prime}, \lambda>0$, and any price vector $\lambda r^{\prime \prime}, \lambda>0$.

The result is immediate from taking convex set combinations in (17) and from scaling vector $r$ in (17).

EXAMPLE 7. Subsidies are used to encourage rotations in the European Union and the United States. To model the effects of such subsidies, let the per acre annual subsidy on the practice be $\phi>0$ and restrict the choice set to $\{\langle C S\rangle,\langle C\rangle,\langle S\rangle\}$. When there are only yield effects due to rotation, when prices such that the grower is indifferent are allocated

to the two-crop rotation, and when the normalization $\alpha_{C \mid C}^{C}=\alpha_{S \mid S}^{S}=0$ is imposed, then the rotation choice is 


$$
\begin{array}{lll}
\langle C S\rangle & \text { if } & \alpha_{C \mid S}^{C} P_{C}+\alpha_{S \mid C}^{S} P_{S}+2 \phi \geq\left|\pi^{C}(r)-\pi^{S}(r)\right| ; \\
\langle C\rangle & \text { if } & \pi^{C}(r)-\pi^{S}(r)>\alpha_{C \mid S}^{C} P_{C}+\alpha_{S \mid C}^{S} P_{S}+2 \phi ; \\
\langle S\rangle & \text { if } & \pi^{S}(r)-\pi^{C}(r)>\alpha_{C \mid S}^{C} P_{C}+\alpha_{S \mid C}^{S} P_{S}+2 \phi .
\end{array}
$$

Figure 3 depicts the regions. When $\phi=0$, then the (positive) prices that support $\alpha_{C \mid S}^{C} P_{C}+\alpha_{S \mid C}^{S} P_{S} \geq\left|\pi^{C}(r)-\pi^{S}(r)\right|$ are in the wedge between two positively sloped rays from the origin. ${ }^{6}$ This is an illustration of Result 4 . Without a subsidy, the convex combination of any two points in the cone labeled $\langle C S\rangle$ must also be in the cone. Picking any point in the cone, all points on the ray from the origin and through it must also be in the cone. The subsidy shifts these rays in a parallel manner so as to expand the price set supporting $\langle C S\rangle$. When a subsidy is employed, then the convex property still applies but the ray property fails.

Returning to equation (17), let $\mathscr{R}$ be the set of permutations on $R_{1}$. So for $R_{1}=\langle A B C\rangle$, the set is $\mathcal{R}=\{\langle A B C\rangle,\langle A C B\rangle\}$ while for $R_{1}=\langle A A A B B B B\rangle$, then $\mathscr{R}=\{\langle A A A B B B B\rangle,\langle A A B A B B B\rangle,\langle A A B B A B B\rangle,\langle A A B B B A B\rangle,\langle A B A B A B B\rangle\}$. Choose weightings $\lambda_{R}, R \in \mathscr{R}$, on the unit simplex for each rotation in $\mathscr{R}$ and generalize (17) to

$$
\sum_{R \in \mathcal{R}} \lambda_{R}\left[V(R)-V\left(R_{1}\right)\right]=\frac{1}{\left|R_{1}\right|} \sum_{t=1}^{h} r_{t} \sum_{u_{j} \in R_{1}}\left(\sum_{R \in \mathcal{R}} \lambda_{R} \alpha_{u_{j} \mid L\left(u_{j} ; R\right)}^{t}-\alpha_{u_{j} \mid L\left(u_{j} ; R_{1}\right)}^{t}\right) .
$$

If there exist simplex weightings $\lambda_{R}, R \in \mathscr{R}$ such that $\sum_{u_{j} \in R_{1}}\left(\sum_{R \in \mathcal{R}} \lambda_{R} \alpha_{u_{j} \mid L\left(u_{j} ; R\right)}^{t}-\alpha_{u_{j} \mid L\left(u_{j} ; R_{1}\right)}^{t}\right) \geq 0 \forall t \in \Omega_{h}$, then a strategy that dominates $R_{1}$ on all land is to sow the land in proportions $\lambda_{R}$ for each $R \in \mathcal{R}$. Thus a price-free condition on carry-over parameters for domination is

RESULT 5. (Switching principle) $R_{1}$ is weakly dominated whenever there exist simplex weightings $\lambda_{R}, R \in \mathcal{R}$ such that $\sum_{u_{j} \in R_{1}}\left(\sum_{R \in \mathcal{R}} \lambda_{R} \alpha_{u_{j} \mid L\left(u_{j} ; R\right)}^{t}-\alpha_{u_{j} \mid L\left(u_{j} ; R_{1}\right)}^{t}\right) \geq 0 \forall t \in \Omega_{h}$. 


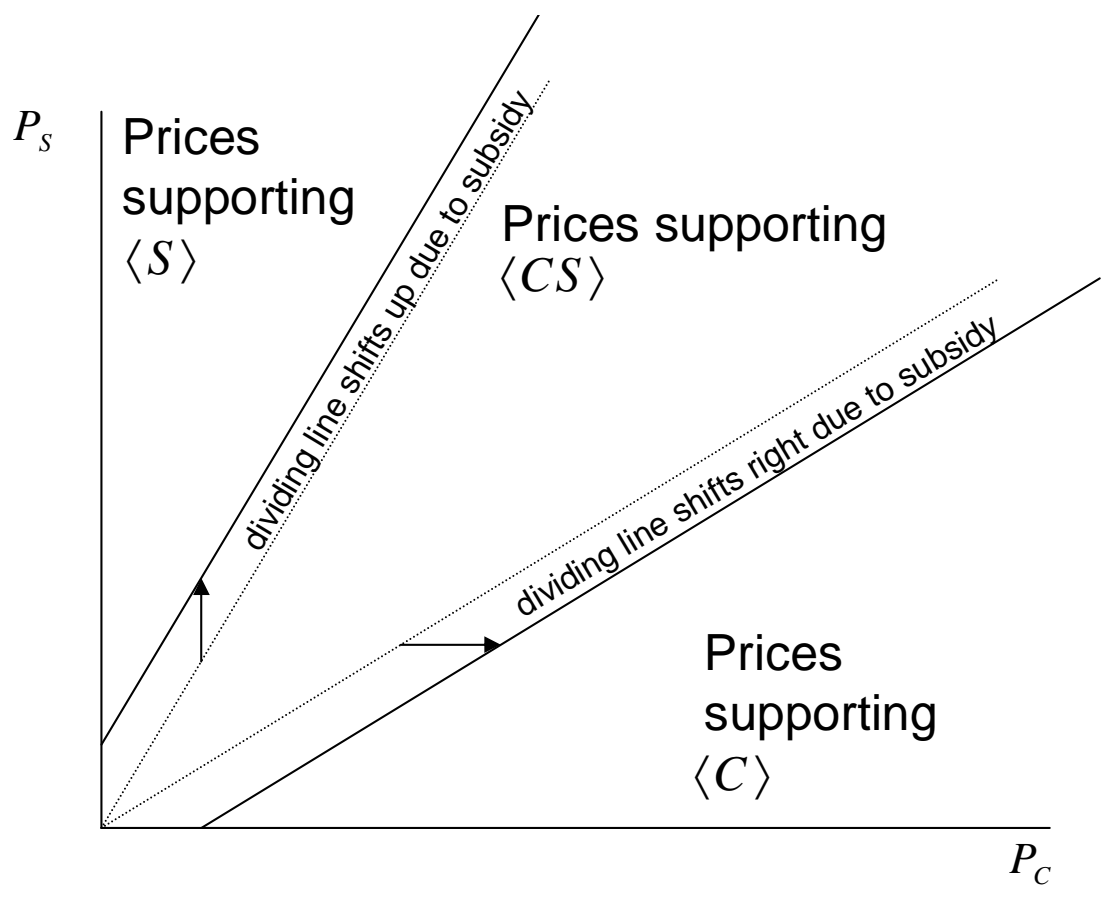

FIGURE 3. Output price space and prices supporting different rotations under a rotation subsidy

In fact, however, the linearity of the model ensures that a portfolio of rotations will not be optimal. Result 5 is not constructive in that it does not identify a rotation that dominates $R_{1}$. The dominating rotations will usually depend upon prices. But if it so happened that the inequality were true for some $\lambda_{R}=1$, for example, return to (17) and suppose that $\sum_{u_{j} \in R_{1}}\left(\alpha_{u_{j} \mid L\left(u_{j} ; R_{2}\right)}^{t}-\alpha_{u_{j} \mid L\left(u_{j} ; R_{1}\right)}^{t}\right) \geq 0$, and then $R$ dominates $R_{1}$ for any positive prices.

EXAMPLE 8. Let $R_{1}=\langle A B C D\rangle$. All remaining permutations are $R_{2}=\langle A D C B\rangle$, $R_{3}=\langle A B D C\rangle, R_{4}=\langle A D B C\rangle, R_{5}=\langle A C B D\rangle$, and $R_{6}=\langle A C D B\rangle$. With one-year memory and $\beta_{i \mid j}^{i \mid k}=\alpha_{i \mid j}^{i}-\alpha_{i \mid k}^{i}$, if there are only yield rotation effects and $R_{1}$ is to dominate all other permutations, then 
$(R 1 R 2) \quad \beta_{A \mid D}^{A \mid B} P_{A}+\beta_{B \mid A}^{B \mid C} P_{B}+\beta_{C \mid B}^{C \mid D} P_{C}+\beta_{D \mid C}^{D \mid A} P_{D} \geq 0 ;$

$(R 1 R 3) \quad \beta_{A \mid D}^{A \mid C} P_{A}+\beta_{C \mid B}^{C \mid D} P_{C}+\beta_{D \mid C}^{D \mid B} P_{D} \geq 0$;

$(R 1 R 4) \quad \beta_{A \mid D}^{A \mid C} P_{A}+\beta_{B \mid A}^{B \mid D} P_{B}+\beta_{D \mid C}^{D \mid A} P_{D} \geq 0$;

$(R 1 R 5) \quad \beta_{B \mid A}^{B \mid C} P_{B}+\beta_{C \mid B}^{C \mid A} P_{C}+\beta_{D \mid C}^{D \mid B} P_{D} \geq 0 ;$

$(R 1 R 6) \quad \beta_{A \mid D}^{A \mid B} P_{A}+\beta_{B \mid A}^{B \mid D} P_{B}+\beta_{C \mid B}^{C \mid A} P_{C} \geq 0$.

For

$\alpha_{A \mid B}^{A}=1, \alpha_{A \mid D}^{A}=1, \alpha_{B \mid C}^{B}=1, \alpha_{B \mid A}^{B}=1, \alpha_{C \mid D}^{C}=0, \alpha_{C \mid B}^{C}=1, \alpha_{D \mid A}^{D}=0, \alpha_{D \mid C}^{D}=1, \alpha_{A \mid C}^{A}=0, \alpha_{D \mid B}^{D}=1$, $\alpha_{B \mid D}^{B}=0, \alpha_{C \mid A}^{C}=0$, then Result 5 applies to ensure a sign on $(R 1 R 2)$ and on each of the other conditions also. In general the whole set of conditions reduces to

$$
P_{C}+P_{D} \geq 0 ; \quad P_{A}+P_{C} \geq 0 ; \quad P_{A}+P_{B}+P_{D} \geq 0 ; \quad P_{C} \geq 0 ; \quad P_{B}+P_{C} \geq 0 ;
$$

and is always satisfied for positive prices because the yield carry-overs for $R_{1}$ are very strong relative to those for the other rotations.

However, if we only change $\alpha_{A \mid D}^{A}=1$ to $\alpha_{A \mid D}^{A}=-1$, then preference over rotations will have to be price dependent. In particular, (21) becomes

$$
P_{C}+P_{D} \geq 2 P_{A} ; \quad P_{C} \geq P_{A} ; \quad P_{B}+P_{D} \geq P_{A} ; \quad P_{C} \geq 0 ; \quad P_{B}+P_{C} \geq 2 P_{A} .
$$

Without searching on the interior of the simplex, it is clear that rotation $\langle A C B D\rangle$ may be removed from further consideration regardless of the level of (positive) prices. We may continue looking for weights that support dominance of $R_{1}$ without needing to include $R_{5}$ in the calculations.

\section{Risk Aversion Effect}

Among the more widely cited motives for use of rotation strategies is risk diversification. We will investigate how rotation carry-over effects interact with diversification effects under the expected utility framework and one-year memory when the choice set is $\{\langle A\rangle,\langle B\rangle,\langle A B\rangle\}$. Harvest output prices are the random variables $\tilde{P}_{A}$ and $\tilde{P}_{B}$, where we will specify distribution assumptions shortly. Were a grower's whole farm devoted to $A$ 
(or $B)$, then output would be $\bar{q}_{A}\left(\bar{q}_{B}\right)$ and costs would be $c_{A}\left(c_{B}\right)$. The fraction of total area devoted to $A$ is $\rho \in[0,1]$.

Rotation effects are confined to yield effects, these being $\alpha_{A \mid B}^{A}$ and $\alpha_{B \mid A}^{B}$ as defined previously. $A A$ and $B B$ carry-over effects are normalized to zero. If $\rho \in(0,0.5)$, then $A$ will always follow $B$ whenever $\alpha_{A \mid B}^{A}>0$ and $\alpha_{B \mid A}^{B}>0$, land will never switch use crop-tocrop whenever $\alpha_{A \mid B}^{A}<0$ and $\alpha_{B \mid A}^{B}<0$, and we cannot be sure of strategy allocation choices when the inequalities differ in direction. Assume that the carryover effects are positive, $\alpha_{A \mid B}^{A}>0$ and $\alpha_{B \mid A}^{B}>0$, so that the stochastic harvest-date payoff is

$$
\Gamma(\rho)=\left(\tilde{P}_{A} \bar{q}_{A}-c_{A}\right) \rho+\left(\tilde{P}_{B} \bar{q}_{B}-c_{B}\right)(1-\rho)+\min [\rho, 1-\rho]\left(\tilde{P}_{A} \alpha_{A \mid B}^{A}+\tilde{P}_{B} \alpha_{B \mid A}^{B}\right) .
$$

The increasing and concave utility function is $U[\Gamma(\rho)]$, the expectation operator over harvest prices is $E\{\bullet\}$, and the planting date objective function is

$$
V(R)=\max _{\rho \in[0,1]} E\{U[\Gamma(\rho)]\}
$$

It is convenient to break the problem in two, writing

$$
\begin{aligned}
& V(R)=\max \{V(R ;[0,0.5]), V(R ;[0.5,1])\} ; \\
& V(R ;[0,0.5])=\max _{\rho \in[0,0.5]} E\left\{U\left[\left(\tilde{P}_{A} \bar{q}_{A}-c_{A}+\tilde{P}_{A} \alpha_{A \mid B}^{A}+\tilde{P}_{B} \alpha_{B \mid A}^{B}\right) \rho+\left(\tilde{P}_{B} \bar{q}_{B}-c_{B}\right)(1-\rho)\right]\right\} ; \\
& V(R ;[0.5,1])=\max _{\rho \in[0.5,0]} E\left\{U\left[\left(\tilde{P}_{A} \bar{q}_{A}-c_{A}\right) \rho+\left(\tilde{P}_{B} \bar{q}_{B}-c_{B}+\tilde{P}_{A} \alpha_{A \mid B}^{A}+\tilde{P}_{B} \alpha_{B \mid A}^{B}\right)(1-\rho)\right]\right\} .
\end{aligned}
$$

We seek to establish conditions such that the optimal cropping strategy is clear. Define $\tilde{Y}=\Gamma(0.5)=0.5 \tilde{P}_{A}\left(\bar{q}_{A}+\alpha_{A \mid B}^{A}\right)+0.5 \tilde{P}_{B}\left(\bar{q}_{B}+\alpha_{B \mid A}^{B}\right)-0.5 c_{A}-0.5 c_{B}, \tilde{X}=\Gamma(0)=\tilde{P}_{B} \bar{q}_{B}-c_{B}$, and $\tilde{Z}=\Gamma(1)=\tilde{P}_{A} \bar{q}_{A}-c_{A}$, and then remove the action constraints:

$$
\begin{gathered}
W(R ;[0,0.5])=\max _{\rho_{i}} E\left\{U\left[\tilde{X} \rho_{i}+\tilde{Y}\left(1-\rho_{i}\right)\right]\right\} \\
W(R ;[0.5,1])=\max _{\rho_{i i}} E\left\{U\left[\tilde{Y} \rho_{i i}+\tilde{Z}\left(1-\rho_{i i}\right)\right]\right\} .
\end{gathered}
$$

The first-order conditions for the unconstrained problems are 


$$
\begin{array}{cc}
W(R ;[0,0.5]): & E\left\{U^{\prime}\left[\tilde{X} \rho_{i}+\tilde{Y}\left(1-\rho_{i}\right)\right](\tilde{X}-\tilde{Y})\right\}=0 \\
W(R ;[0.5,1]): & E\left\{U^{\prime}\left[\tilde{Y} \rho_{i i}+\tilde{Z}\left(1-\rho_{i i}\right)\right](\tilde{Y}-\tilde{Z})\right\}=0
\end{array}
$$

but it is the corner solutions for the constrained problems that are of interest. In this regard, two definitions are in order. Define the harmonic mean of, say, $\tilde{Y}$, as $H(\tilde{Y})=E\left\{U^{\prime}[\tilde{Y}] \tilde{Y}\right\} / E\left\{U^{\prime}[\tilde{Y}]\right\}$, as in Kijima 1997 or in McEntire 1984. Then, define as follows. ${ }^{7}$

DefinITION 3. (Shaked and Shanthikumar 1994, p. 118) Random variables $\tilde{P}_{A}$ and $\tilde{P}_{B}$ are said to be associated if $E\left\{G^{1}\left(\tilde{P}_{A}, \tilde{P}_{B}\right) G^{2}\left(\tilde{P}_{A}, \tilde{P}_{B}\right)\right\} \geq E\left\{G^{1}\left(\tilde{P}_{A}, \tilde{P}_{B}\right)\right\} E\left\{G^{2}\left(\tilde{P}_{A}, \tilde{P}_{B}\right)\right\}$ for all non-decreasing functions $G^{1}\left(\tilde{P}_{A}, \tilde{P}_{B}\right)$ and $G^{2}\left(\tilde{P}_{A}, \tilde{P}_{B}\right)$ such that the expectations exist.

This is a generalized form of correlation, and it does require that $\tilde{P}_{A}$ bear a positive linear correlation with $\tilde{P}_{B}$. Positive association between these random variables is reasonable because commodity prices tend to covary positively. In the use we put the concept to, association ensures that diversification is not so effective that a mildly risk-averse individual could be coaxed out of the decision under risk neutrality into some of an investment that accrues large losses in expectation. Concerning corner and interior solutions, some analysis demonstrates that the situations in which $\rho_{i}^{*} \in(0,1)$ and $\rho_{i i}^{*} \in(0,1)$, where $\rho_{i}^{*} \in(0,1)$ and $\rho_{i i}^{*} \leq 0$, and also in which $\rho_{i}^{*} \geq 1$ and $\rho_{i i}^{*} \in(0,1)$ may all be ruled out under association and positive rotation carry-over effects. There are six remaining possible cases, and only four are essentially distinct. The distinct cases are as follows.

Case 1: $\rho_{i}^{*} \leq 0, \rho_{i i}^{*} \geq 1$. From (27), this means that $H(\tilde{Y}) \geq E\left\{U^{\prime}[\tilde{Y}] \tilde{X}\right\} / E\left\{U^{\prime}[\tilde{Y}]\right\}$ and $H(\tilde{Y}) \geq E\left\{U^{\prime}[\tilde{Y}] \tilde{Z}\right\} / E\left\{U^{\prime}[\tilde{Y}]\right\}$. By the association property on $\tilde{P}_{A}$ and $\tilde{P}_{B}$, both of these conditions are certainly satisfied whenever $H(\tilde{Y}) \geq \max [E\{\tilde{X}\}, E\{\tilde{Z}\}]$. Since the problems in (26) are convex in the choice variable, $H(\tilde{Y}) \geq \max [E\{\tilde{X}\}, E\{\tilde{Z}\}]$ ensures that the acreage allocation decision is entirely to $\langle A B\rangle$. 
Case 2: $\rho_{i}^{*} \geq 1, \rho_{i i}^{*} \leq 0$. This occurs when $E\left\{U^{\prime}[\tilde{Z}] \tilde{Y}\right\} / E\left\{U^{\prime}[\tilde{Z}]\right\} \leq H(\tilde{Z})$ and $H(\tilde{X}) \geq E\left\{U^{\prime}[\tilde{X}] \tilde{Y}\right\} / E\left\{U^{\prime}[\tilde{X}]\right\}$. In this case, monoculture is assured. Due to association, both of these conditions apply if $\min [H(\tilde{X}), H(\tilde{Z})] \geq E\{\tilde{Y}\}$. The crop that is grown is the one that maximizes expected utility under monoculture.

Case 3: $\rho_{i}^{*} \geq 1, \rho_{i i}^{*} \geq 1$ (symmetrically: $\rho_{i}^{*} \leq 0, \rho_{i i}^{*} \leq 0$ ). In this case, $\tilde{X}$ dominates any convex combination of $\tilde{X}$ and $\tilde{Y}$ while $\tilde{Y}$ dominates any convex combination of $\tilde{Y}$ and $\tilde{Z}$ so that all acres are sown under $\langle B\rangle$. The conditions may be written as $H(\tilde{X}) \geq E\left\{U^{\prime}[\tilde{X}] \tilde{Y}\right\} / E\left\{U^{\prime}[\tilde{X}]\right\}$ and $H(\tilde{Y}) \geq E\left\{U^{\prime}[\tilde{Y}] \tilde{Z}\right\} / E\left\{U^{\prime}[\tilde{Y}]\right\}$. Under association, the pair of inequalities $H(\tilde{X}) \geq E\{\tilde{Y}\}$ and $H(\tilde{Y}) \geq E\{\tilde{Z}\}$ ensure that monoculture under $B$ is chosen. In the symmetric case, sufficient conditions that monoculture under $A$ is chosen are that association applies together with $H(\tilde{Z}) \geq E\{\tilde{Y}\}$ and $H(\tilde{Y}) \geq E\{\tilde{X}\}$.

Case 4: $\rho_{i}^{*} \in(0,1), \rho_{i i}^{*} \geq 1$ (symmetrically: $\rho_{i}^{*} \leq 0, \rho_{i i}^{*} \in(0,1)$ ). Given the solution to the first problem, we can assert that a convex combination of $\langle B\rangle$ and $\langle A B\rangle$ is preferred to $\langle A B\rangle$. The solution to the second problem shows that $\langle A B\rangle$ is preferred to $\langle A\rangle$ or any interior convex combination of $\langle A\rangle$ and $\langle A B\rangle$. Thus, the solution is to choose a convex combination of $\langle B\rangle$ and $\langle A B\rangle$. Under the symmetric case, it is optimal to choose a convex combination of $\langle A\rangle$ and $\langle A B\rangle$.

Function $H(\cdot)$ is just the standard expectation when the utility function describes risk neutrality and then the decisions reduce to those described earlier in this paper. Clearly, an understanding of how the degree of risk aversion affects function $H(\cdot)$ would be useful. For the pair of utility functions $\hat{U}(\cdot)$ and $\hat{U}(\cdot)$ and for random variable $\tilde{\theta}$, write the respective harmonic means as $\hat{H}(\tilde{\theta})=E\left\{\hat{U}^{\prime}(\tilde{\theta}) \tilde{\theta}\right\} / E\left\{\hat{U}^{\prime}(\tilde{\theta})\right\}$ and $\hat{H}(\tilde{\theta})=E\left\{\hat{U}^{\prime}(\tilde{\theta}) \tilde{\theta}\right\} / E\left\{\hat{U}^{\prime}(\tilde{\theta})\right\}$. Find the difference, insert the irrelevant parameter $\bar{\theta}$, and rearrange to obtain 


$$
\hat{H}(\tilde{\theta})-\hat{H}(\tilde{\theta})=\frac{1}{E\left\{\hat{U}^{\prime}(\tilde{\theta})\right\}} E\left\{\widehat{U}^{\prime}(\tilde{\theta})\left[\frac{\hat{U}^{\prime}(\tilde{\theta})}{\hat{U}^{\prime}(\tilde{\theta})}-\frac{E\left\{\hat{U}^{\prime}(\tilde{\theta})\right\}}{E\left\{\hat{U}^{\prime}(\tilde{\theta})\right\}}\right](\tilde{\theta}-\bar{\theta})\right\} .
$$

If $\hat{U}^{\prime}(\tilde{\theta}) / \hat{U}^{\prime}(\tilde{\theta})$ is an increasing function, then $\hat{U}^{\prime}(\tilde{\theta}) / \hat{U}^{\prime}(\tilde{\theta})-E\left\{\hat{U}^{\prime}(\tilde{\theta})\right\} / E\left\{\hat{U}^{\prime}(\tilde{\theta})\right\}$

crosses from negative value to positive value at some $\tilde{\theta}$ value, and we set this value as $\bar{\theta}$. Since the product of terms inside the large parentheses in (28) is always non-negative, it follows that $\hat{H}(\tilde{\theta}) \geq \hat{H}(\tilde{\theta})$ whenever $\hat{U}^{\prime}(\tilde{\theta}) / \hat{U}^{\prime}(\tilde{\theta})$ is increasing, that is, $-\hat{U}^{\prime \prime}(\tilde{\theta}) / \hat{U}^{\prime}(\tilde{\theta}) \leq-\hat{U}^{\prime \prime}(\tilde{\theta}) / \hat{U}^{\prime}(\tilde{\theta})$. So we can be sure that $H(\tilde{\theta})$ is not much smaller than $E\{\tilde{\theta}\}$ when the coefficient of risk aversion on the utility function entering $H(\tilde{\theta})$ is negative but close to zero.

This observation allows us to summarize the earlier case analysis. Given statistical association between variables $\tilde{P}_{A}$ and $\tilde{P}_{B}$, if a risk-neutral individual has strict preference among $\{\langle A\rangle,\langle B\rangle,\langle A B\rangle\}$, then the introduction of a small level of risk aversion will not change the preference. As the level of risk aversion increases, though, a switch to mixing monoculture with rotation (Case 4) or a switch to another choice among $\{\langle A\rangle,\langle B\rangle,\langle A B\rangle\}$ can occur. Thus, risk aversion can explain the rotation $\langle C C S\rangle$ in Example 2 even when one-year memory applies.

\section{Time Rationing}

A further motive for use of rotations is workload management. The argument is that different crops have different seasonal workload requirements, and so growing a mix of crops could be more efficient than specialization. The motive concerns competing demands for resources (time, versatile machinery, working capital, etc.) and not temporal spillovers in crop productivity. To evaluate the argument, we ignore risk and introduce a seasonal labor cost function but otherwise adopt the model in the previous section.

There are $J$ seasons in the year, and the seasons are denoted as $j \in \Omega_{J}$. The cost of hiring $T$ units of labor in any season is $C[T]$, a twice continuously differentiable, increasing, and convex function. One acre can be allocated across crops $\{A, B\}$. The $i$ th crop profit per acre before time costs is $\pi^{i}$, and land share $\rho$ is devoted to crop $A$. The 
time requirement of the $i$ th crop in season $j$ is $t_{i, j}$. Annual profit is

$$
\begin{aligned}
& V(\rho)=\pi^{A} \rho+\pi^{B}(1-\rho)+\min [\rho, 1-\rho]\left(P_{A} \alpha_{A \mid B}^{A}+P_{B} \alpha_{B \mid A}^{B}\right)-\sum_{j \in \Omega_{J}} C\left[T_{j}(\rho)\right] \\
& T_{j}(\rho)=t_{A, j} \rho+t_{B, j}(1-\rho) ; \quad \rho \geq 0 ;
\end{aligned}
$$

Breaking the optimization problem in two, write

$$
\begin{aligned}
& \text { (30i) } \max _{\rho \in[0,0.5]}\left(\pi^{A}+P_{A} \alpha_{A \mid B}^{A}+P_{B} \alpha_{B \mid A}^{B}\right) \rho+\pi^{B}(1-\rho)-\sum_{j \in \Omega_{J}} C\left[T_{j}(\rho)\right] \\
& \text { (30ii) } \max _{\rho \in[0.5,1]} \pi^{A} \rho+\left(\pi^{B}+P_{B} \bar{q}_{B}+P_{A} \alpha_{A \mid B}^{A}\right)(1-\rho)-\sum_{j \in \Omega_{J}} C\left[T_{j}(\rho)\right] .
\end{aligned}
$$

At this point, the analysis can proceed much as for the study of risk aversion. Conditions can be specified such that each of Cases 1 through 4 occurs.

We close with a point on the role of correlation among crop labor demands. To make explicit how labor requirement schedules affect the optimal solution, suppose that $C\left[T_{j}\right]=0.5 \kappa T_{j}^{2}, \kappa>0$. Using notation $\sigma_{i, k}=J^{-1} \sum_{j \in \Omega_{j}} t_{i, j} t_{k, j}$, suppose that $\pi^{A}=\pi^{B}$ and $\alpha_{A \mid B}^{A}=\alpha_{B \mid A}^{B}=0$ so that rotation effects are absent and there is no bias in baseline profits. Then the two sub-problems merge so that $\rho_{i i}^{*}=\rho_{i i}^{*}\left[\equiv \rho^{*}\right]$ and

$$
\rho^{*}=\frac{\sigma_{B, B}-\sigma_{A, B}}{\sigma_{A, A}+\sigma_{B, B}-2 \sigma_{A, B}} .
$$

Differentiation with respect to $\sigma_{A, B}$ provides $d \rho^{*} / d \sigma_{A, B} \stackrel{\text { sign }}{=} \sigma_{B, B}-\sigma_{A, A} \stackrel{\text { sign }}{=} \rho^{*}-0.5$. If two enterprises give equal profits, apart from seasonal labor costs, that are increasing, convex and quadratic, then an increase in the correlation between the labor needs of the two pushes the optimal allocation decision toward the one already favored. Note that the corner solution $\rho^{*}=1$ is supported whenever $\sigma_{A, B} \geq \sigma_{A, A}$ and this is possible; the Schwartz inequality (Rudin 1976) only requires that $\sigma_{A, A} \sigma_{B, B}>\sigma_{A, B}^{2}$ for variables that are not perfectly linearly correlated. If $\sigma_{A, B} \geq \sigma_{A, A}$ then $\sigma_{B, B}$ is large. Crop $B$ provides little in the way of labor requirement diversification so that the labor costs of this enterprise are prohibitive. In general equilibrium, and if this farm's experience is typical, one might 
expect an increase in $P_{B}$ such that some growers are willing to meet demand. If $\sigma_{A, B} \leq 0$ (a violation of statistical association), then there will be no corner solutions to (31) because the crop mix is very effective at stabilizing labor demand while other economic parameters are not such that they promote specialization.

\section{Conclusion}

Recognizing the importance of crop rotation for private profit and public policy, the intent of this paper has been to investigate some economics behind the choice. Our main model has provided some rules of thumb for choosing among rotations. General insights are that rotation carry-over can support quite involved rotations only if monoculture profits are narrowly dispersed and carry-over effects persist for several years. One exception is the case in which substantial fixed costs are incurred to initiate a crop while carry-over fertility effects on a secondary crop accumulate to a significant level over several years. Then a dominant crop may be rotated with occasional planting of the secondary crop. Things are not quite so straightforward under risk aversion in the presence of uncertainty because rotation and diversification effects can trade off such that mixing monoculture with rotations may occur. We also show that monoculture and mixing monoculture with rotations can be motivated by time rationing among crops.

We have noted in passing several other motives for choosing monoculture but have not developed the arguments. Nor have we engaged in any empirical studies to discriminate between motives. These are the logical next steps. The inavailability of commercial cropping choice data attached to relevant farm-level technology data may have been responsible in part for a paucity of research on the economics of rotation decisions to date. ${ }^{8}$ Governmental data efforts in recent years, together with technical advances in the gathering and analysis of information, hold promise for discerning the relative importance of factors in determining rotation choices. 


\section{Endnotes}

1. For the Upper Mississippi River Basin, Wu et al. (2004) found subsidies on rotations to have only a weak effect in altering practices to reduce run-off pollution.

2. Throughout, we assume that one crop is grown per year. This is a convenience to economize on the use of notation.

3. The $U$ identifies just one $u_{i} u_{i}$ sequence in the rotation. A second sequence $u_{i} u_{i}$ in the same rotation is not represented by $U$.

4. For example, nitrogen requirements for crop $A$ may depend upon the two preceding crops. Crop $B$ may have a different root system so that the nitrogen requirement depends only upon the preceding crop.

5. For example,

$\max \left\{V\left(\left\langle u_{1}\right\rangle\right), V\left(\left\langle u_{2}\right\rangle\right)\right\}=\max \left\{V\left(\left\langle u_{2}\right\rangle\right), V\left(\left\langle u_{1}\right\rangle\right)\right\}=\lambda \max \left\{V\left(\left\langle u_{1}\right\rangle\right), V\left(\left\langle u_{2}\right\rangle\right)\right\}+$ $(1-\lambda) \max \left\{V\left(\left\langle u_{2}\right\rangle\right), V\left(\left\langle u_{1}\right\rangle\right)\right\}$

$\geq \max \left\{\lambda V\left(\left\langle u_{1}\right\rangle\right)+(1-\lambda) V\left(\left\langle u_{2}\right\rangle\right), \lambda V\left(\left\langle u_{2}\right\rangle\right)+(1-\lambda) V\left(\left\langle u_{1}\right\rangle\right)\right\}$. Then set $\lambda=1 /|R|=0.5$.

6. Linear homogeneity of the profit function ensures that the partitioning curves are rays.

7. Association is weaker, i.e., less restrictive, than the affiliation assumption that is widely used in auction theory (Shaked and Shanthikumar 1994, p. 254; Milgrom 1989).

8. Innovations in remote imaging allow reliable detection of agricultural subsidy fraud in which planting decisions are misrepresented (Mitchener 2004). 


\section{References}

Batra, S.W.T. 1982. "Biological Control in Agroecosystems.” Science 215(8, January):134-39.

Baumhardt, R.L. 2003. "Dust Bowl Era.” In Encyclopedia of Water Science. Edited by B.A. Stewart and T.A. Howell, pp. 187-191. New York: Marcel Dekker.

Brandon, P.F. 1972. "Cereal Yields on the Sussex Estates of Battle Abbey during the Later Middle Ages." Economic History Review 25(August): 403-20.

Cai, D., M. Kleine, S. Kifle, H-J. Harloff, N.N. Sandal, K.A. Marcker, R.M. Klein-Lankhorst, E.M.J. Salentijn, W. Lange, W.J. Stiekema, U. Wyss, F.M.W. Grundler, and C. Jung. 1997. "Positional Cloning of a Gene for Nematode Resistance in Sugar Beet." Science 275(7, February):832-34.

Camara, O.M., D.L. Young, and H.R. Hinman. 1999. "Economic Case Studies of Eastern Washington and Northern Idaho No-Till Farmers Growing Wheat, Barley, Lentils, and Peas in the 19-22 Inch Precipitation Zone.” Farm Business Management Report EB1886, Washington State University.

Campbell, C.A., R.P. Zentner, H.H. Janzen, and K.E. Bowren. 1990. "Crop Rotation Studies on the Canadian Prairies." Publication No. 1841/E, Research Branch, Agriculture Canada.

Chambers, R.G., and E. Lichtenberg. 1995. "Economics of Sustainable Farming in the Mid-Atlantic." Final report presented to the U.S. Department of Agriculture/Environmental Protection Agency program, Agriculture in Concert with the Environment, Washington, DC.

Collins, R.A., and P.J. Barry. 1986. "Risk Analysis with Single-Index Portfolio Models: An Application to Farm Planning." American Journal of Agricultural Economics 68(February): 152-61.

Cowan, R., and P. Gunby. 1996. "Sprayed to Death: Path Dependence, Lock-In and Pest Control Strategies.” Economic Journal 106(May): 521-42.

Färe, R., and S. Grosskopf. 1996. Intertemporal Production Frontiers: With Dynamic DEA. Boston: Kluwer Academic Publishers.

Froot, K.A., D.S. Scharfstein, and J.C. Stein. 1993. "Risk Management: Coordinating Corporate Investment and Financing Policies." Journal of Finance 48(December 1993): 1629-58.

Heady, E.O. 1951. "Resource and Revenue Relationships in Agricultural Production Control Programs." Review of Economics and Statistics 33(August): 228-40.

Jaenicke, E.C. 2000. "Testing for Intermediate Outputs in Dynamic DEA Models: Accounting for Soil Capital in Rotational Crop Production and Productivity Measures." Journal of Productivity Analysis 14(November): 247-66.

Kijima, M. 1997. "The Generalized Harmonic Mean and a Portfolio Problem with Dependent Assets." Theory and Decision 43(July): 71-87. 
On Monoculture and the Structure of Crop Rotations / 29

Koopmans, T.C., ed. 1951. Activity Analysis of Production and Allocation; Proceedings of a Conference. Monograph No. 13, Cowles Commission for Research in Economics. New York: Wiley.

Lal, R., R.F. Follett, J.M. Kimble, and C.V. Cole. 1999. "Managing U.S. Cropland to Sequester Carbon in Soil." Journal of Soil and Water Conservation 54(Winter): 374-81.

Lewandrowski, J., M. Peters, C. Jones, R. House, M. Sperow, M. Eve, and K. Paustian. 2004. Economics of Sequestering Carbon in the U.S. Agricultural Sector. ERS Technical Bulletin No. 1909, U.S. Department of Agriculture, Economic Research Service. Washington, DC. April.

McEntire, P.L. 1984. "Portfolio Theory for Independent Assets." Management Science 30(August): 952-63.

Milgrom, P. 1989. “Auctions and Bidding: A Primer.” Journal of Economic Perspectives 3(Summer): 3-22.

Miller, M.E. 2003. "An Economic Perspective on Iowa Farm Diversification in the Twenthieth Century." Unpublished creative component for MS degree in economics, Iowa State University.

Mitchener, B. 2004. “EU Harvests Satellite Images.” Wall Street Journal, July 20, p. A9.

Musoke, M.S., and A.L. Olmstead. 1982. "The Rise of the Cotton Industry in California: A Comparative Analysis." Journal of Economic History 42(June) :385-412.

Newell, W.H. 1973. "The Agricultural Revolution in Nineteenth-Century France.” Journal of Economic History 33(December): 697-731.

Pimentel, D., C. Harvey, P. Resosudormo, K. Sinclair, D. Kurz, M. McNair, S. Crist, L. Shpritz, L. Fitton, R. Saffouri, and R. Blair. 1995. "Environmental and Economic Costs of Soil Erosion and Conservation Benefits.” Science 267(24 February): 1117-23.

Plumb, J.H. 1952. "Sir Robert Walpole and Norfolk Husbandry.” Economic History Review 5: 86-89.

Poggi, E.M. 1930. “The German Sugar Beet Industry.” Economic Geography 6(January): 81-93.

Rhode, P.W. 1995. "Learning, Capital Accumulation, and the Transformation of California Agriculture." Journal of Economic History 55(December): 773-800.

Rudin, W. 1976. Principles of Mathematical Analysis, 3rd ed. New York: McGraw-Hill.

Saloutos, T. 1946. "The Spring-Wheat Farmer in a Maturing Economy 1870-1920.” Journal of Economic History 6(November): 173-90.

Shaked, M., and J.G. Shanthikumar. 1994. Stochastic Orders and their Applications. San Diego: Academic Press.

Sharpley, A.N., and J.R. Williams, eds. 1990. EPIC-Erosion/Productivity Impact Calculator: 1. Model Documentation. Technical Bulletin No. 1768, U.S. Department of Agriculture. Washington, DC. April.

Sokoloff, K.L., and D. Dollar. 1997. "Agricultural Seasonality and the Organization of Manufacturing in Early Industrial Economies: The Contrast Between England and the United States." Journal of Economic History 55(June): 288-321.

Stilgenbauer, F.A. 1927. “The Michigan Sugar Beet Industry.” Economic Geography 3(October): 486-506. 
Thomas, A. 2003. “A Dynamic Model of On-Farm Integrated Nitrogen Management." European Review of Agricultural Economics 30(December): 439-60.

Timmer, C.P. 1969. "The Turnip, the New Husbandry, and the English Agricultural Revolution.” Quarterly Journal of Economics 83(August): 375-95.

Wang, E., W.L. Harman, J.R. Williams, and C. Xu. 2002. "Simulated Effects of Crop Rotations and Residue Management on Wind Erosion in Wuchuan, West-Central Inner Mongolia, China." Journal of Environmental Quality 31: 1240-47.

Whatley, W.C. 1987. "Southern Agrarian Labor Contracts as Impediments to Cotton Mechanization." Journal of Economic History 47(March): 45-70.

Windish, L.G. 1981. The Soybean Pioneers: Trailblazers ... Crusaders ... Missionaries. Galva, IL: Leo G. Windish.

Wu, J., R.M. Adams, C.L. Kling, and K. Tanaka. 2004. "From Microlevel Decisions to Landscape Changes: An Assessment of Agricultural Conservation Policies." American Journal of Agricultural Economics 86(February 2004): 26-41. 